\title{
Application of Neutron Radiography to Image Flow Phenomena in Porous Media
}

\author{
J. K. Jasti and H. S. Fogler \\ Dept. of Chemical Engineering, The University of Michigan, Ann Arbor, MI 48109
}

\begin{abstract}
Neutron transmission imaging is a technique ideally suited for imaging various flow phenomena in consolidated porous media because the porous matrix is virtually transparent to thermal neutrons. Hydrogen-containing fluids, however, provide a contrast in the image due to their large thermal neutron cross-sections. This technique has uncovered new phenomena in the study of dissolution and precipitation in porous media. Neutron images of acid-etched porous patterns show that the degree of branching, and the tortuosity of the etched patterns depend on competing effects of acid flow rate, dissolution rate and precipitation rate of the reaction products. The fine structure of etched pathways show that they can propagate against the direction of flow, a phenomenon not previously observed. In a second study, realtime neutron transmission imaging was used to visualize miscible tracer dispersion. It was shown how quantitative information such as the in-situ spatial distributions of the tracer concentrations can be obtained with this technique.
\end{abstract}

\section{Introduction}

Flow in porous media is a challenging scientific problem having great technological importance. Lack of detailed structural information at the microscale and the mathematical difficulties caused by the randomness of the porous medium make the application of first principles very difficult. Hence, flow visualization is a valuable tool in understanding various flow phenomena in porous media. Historically, several techniques have been used to obtain spatial information during a typical core-flood experiment (Wellington and Vinegar, 1987). Some of the methods include: transparent models, microwave absorption, nuclear magnetic resonance (NMR), $X$-ray and gamma-ray attenuation, radio isotope injection, and magnetic susceptibility. Most of these methods provide only two-dimensional information and therefore, are limited in their use.

Recently, there has been an intensified effort in utilizing three-dimensional imaging methods to study various flow phenomena in porous media. The two prominent systems are based on X-ray and NMR computerized tomography (CT), and they were originally developed for medical applications. While these systems have been shown to be powerful, they have some limitations. The X-ray CT system requires the use of excessive dopants to distinguish fluids, whereas the NMR system is limited to porous media having very small concentrations of paramagnetic ions. It should be pointed out that the advantages of the two systems far outweigh the limitations mentioned here. For most consolidated porous media, these limitations are not severe enough to discourage the use of these imaging systems.

We describe a thermal-neutron-based CT system as an alternative three-dimensional imaging system to NMR and $X$ ray $C T$ systems for visualizing flow phenomena in porous media. It was originally developed for industrial diagnostics and offers complimentary information when compared with an X-ray system. In a manner similar to the X-ray CT, the neutron imaging system can be operated in a radiograph mode to obtain two-dimensional transmission images having a high degree of spatial resolution or in the real-time mode to image dynamic events having a temporal resolution of the order of $1 / 32$ of a second. Both systems can be used in conjunction with computerized tomography for three-dimensional imaging. Reactive dissolution of carbonates and miscible tracer flow in porous media are used as examples to demonstrate the feasibility of this technique.

\section{Description of the Facility}

Figures 1 and 2 show two different methods of imaging (Barton and von der Hardt, 1981; Hawkesworth and Walker, 1983). MW nuclear reactor provides a two-dimensional col- 


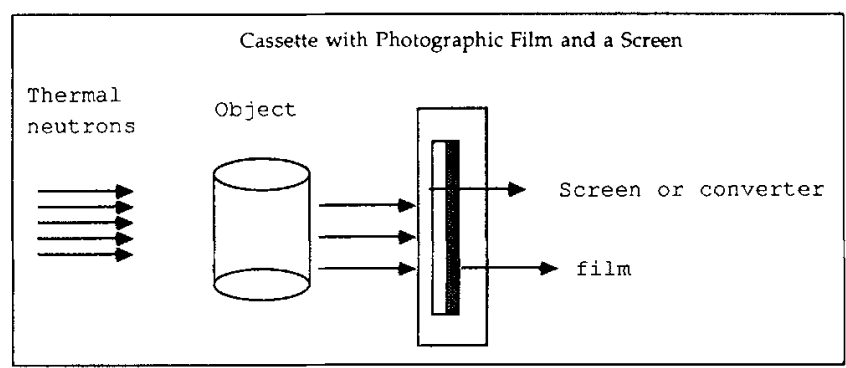

Figure 1. Film neutron radiographic system.

limated thermal neutron beam at a flux of $10^{6} / \mathrm{cm}^{2} / \mathrm{s}$. The object to be imaged is placed in the path of the neutron beam, and neutron flux passing through the object is measured. In the film radiography method (Figure 1), the neutron flux is recorded onto a photographic film. Since thermal neutrons cannot directly expose the film, a screen is used to absorb thermal neutrons and to generate a proportional amount of actinic radiation. Gadolinium oxysulfide and vapor-deposited gadolinium powder are used as screens in this study, and they require exposure times of $1 \mathrm{~min}$ and $20 \mathrm{~min}$, respectively. The photographic film can be processed using standard procedures. Longer exposure times limit the use of the film method to imaging static processes. The advantages of the film method are its simplicity and high degree of spatial resolution of the images.

The real-time image system in Figure 2 can be used to record dynamic events with time resolution close to $1 / 32$ of a second. The image intensifier coupled with a Vidicon camera acts as a neutron camera. The intensifier has a gadolinium oxysulfide phosphor which converts neutrons into light. The light output generated by the phosphor screen is amplified a million-fold so that it can be processed by a video system. A Vidicon camera, a high-resolution tape recorder, and TV monitors constitute the video system. The video signal can also be digitized into a rectangular array $(640 \times 480)$ of pixels using a PC-based imaging system. While this system cannot match the spatial resolution of the film radiography method, it can be used to record dynamic events.

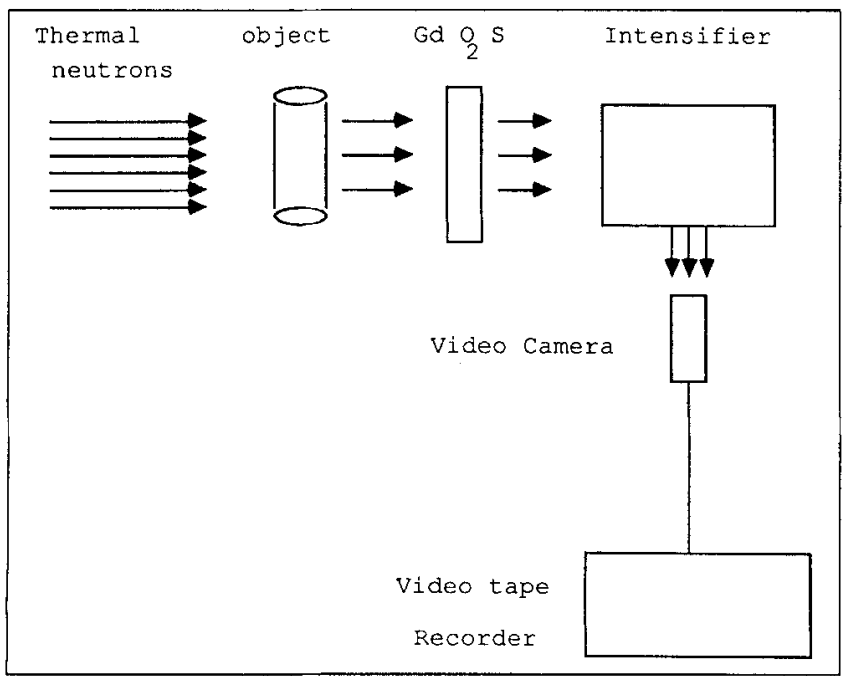

Figure 2. Real-time neutron imaging facility.

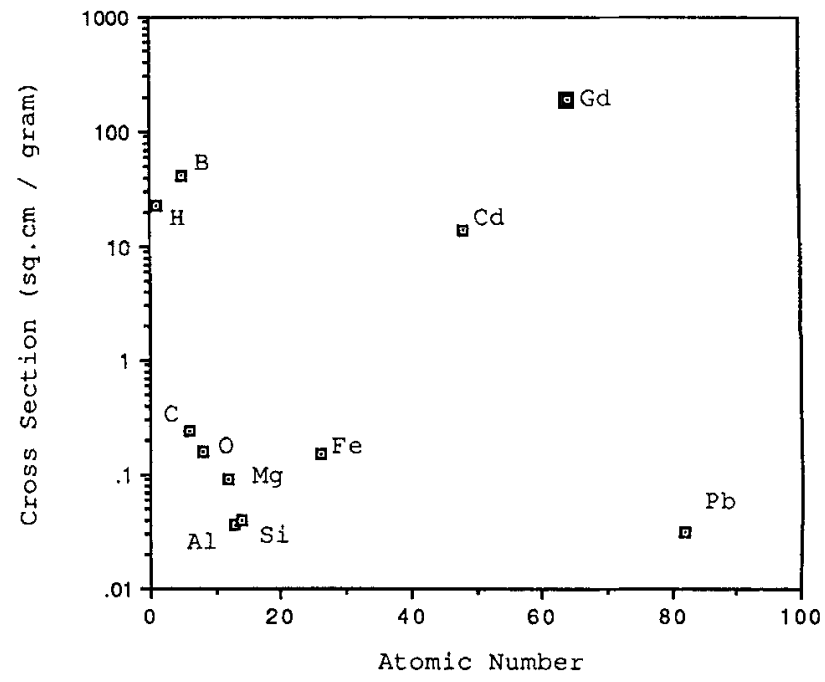

Figure 3. Thermal neutron cross-sections of some elements.

Since both systems image thermal neutron transmission characteristics of an object, the ability to image certain features in a porous medium depends on the thermal neutron crosssections. Cross-sections for some elements are plotted as a function of the atomic number in Figure 3. One notes that there is no correlation between the cross-section and the atomic number. Very few elements such as gadolinium, cadmium, boron, and hydrogen possess large cross-sections. Because naturally occurring porous media contain elements with small neutron cross-sections, they are virtually transparent to thermal neutrons and consequently, thermal neutrons are ideally suited for imaging the flow of hydrogen containing fluids.

Figure 4 shows how quantitative information can be obtained from the neutron transmission images using neutron attenuation properties. The transmission of neutrons through a sample may be expressed by the following exponential relation between the neutron flux and distance:

$$
\frac{I}{I_{o}}=\exp (-\mu \rho x)
$$

where

$I=$ thermal neutron flux passing through the object

$x=$ thickness of the object

$\mu=$ thermal neutron cross-section of the object

$\rho=$ density of the object

$I_{o}=$ incident thermal neutron flux

The product of the density and the cross-section is called a linear attenuation coefficient. The intensity of neutrons passing

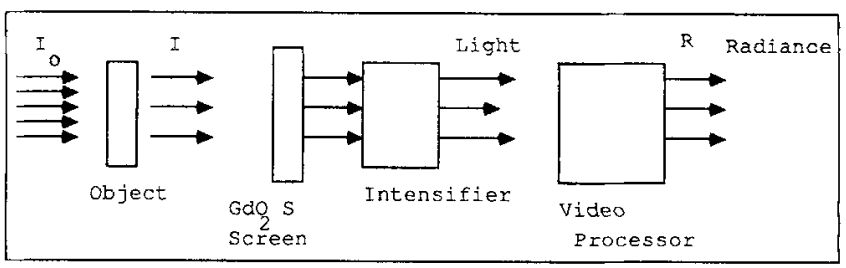

Figure 4. Digitization process used for obtaining quantitative information. 
through the object is converted into a proportional light intensity either by the film radiography method or the real-time imaging method. The light images are then digitized onto a two-dimensional grid using an eight-bit image processing system. Each pixel on the digitized mesh can have a brightness in the range of 0 to 255 and this value is called radiance unit, $R$. Since radiance units vary linearly with the neutron flux, Eq. 1 can be rewritten in terms of radiance units:

$$
\frac{R}{R_{0}}=\exp (-\mu \rho x)
$$

If the object contains several components, the exponent in the above equation can be modified as the weighted average of various components (Barton and von der Hardt, 1981).

$$
[\mu \rho x]_{\text {mixture }}=\sum_{i} w_{i}[\mu \rho x]_{i}
$$

The cross-sections of the components can be adjusted so that the component to be imaged provides a high contrast in the transmission image. Table 1 shows neutron transmission characteristics of some materials commonly used to image flow in porous media. The cross-sections for these materials have been tabulated by Barton and van der Hardt. Since most of the oil-bearing formations are either sandstones or carbonates, they are ideal for imaging the flow of fluids containing hydrogen. The large difference between the cross-sections of the porous media and fluids containing hydrogen results in a high contrast in neutron images. It is also possible to image the flow of two liquids by replacing hydrogen in one of the fluids with deuterium, because cross-sections for water and deuterium oxide differ significantly: $3.48 \mathrm{~cm}^{2} / \mathrm{g}$ for water and 0.41 $\mathrm{cm}^{2} / \mathrm{g}$ for deuterium oxide. Similarly, a high contrast in images for gas-liquid flows can be obtained because of small crosssections of gases. In addition, we can use a large cross-section material, such as Woods alloy, to image porosity patterns etched during the reactive dissolution of porous media.

Quantitative information such as the spatial distribution of a component in porous media can be obtained from the neutron transmission images. A tracer having a large neutron crosssection can be used to image miscible tracer dispersion. An image of the porous matrix initially saturated with the solvent is used as a mask, and its contribution is subtracted from the rest of the images. The net result only shows the images of the tracer. In-situ tracer concentration profiles can be determined from the following exponential relation:

$$
\frac{R}{R_{0}}=\exp (-\mu \phi x C)
$$

\footnotetext{
where

$R=$ radiance unit recorded by the video system

$R_{0}=$ radiance unit of the incident neutron beam

$\phi=$ porosity of the rock matrix

$C=$ concentration of the tracer
}

In practice, the relationship between the radiance and tracer concentration deviates from the exponential relation due to artifacts caused by the nonuniformity of the incident neutron beam, fluctuations in neutron flux during the course of the experiment, changes in the neutron energy spectrum with po-
Table 1. Thermal Neutron Transmission Characteristics of Common Compounds Relevant to Flow in Porous Media

\begin{tabular}{ll}
\hline Transparent & Opaque \\
\hline $\mathrm{SiO}_{2}$ & $\mathrm{H}_{2} \mathrm{O}$ \\
$\mathrm{CaCO}_{3}$ & Oil \\
$\mathrm{Al}_{2} \mathrm{O}_{3}$ & Sodium Borate \\
$\mathrm{D}_{2} \mathrm{O}$ & Woods Metal \\
$\mathrm{Halocarbon}$ Oils & Gadolinium Chloride \\
$\mathrm{CO}_{2}$ & - \\
$\mathrm{N}_{2}$ & - \\
\hline
\end{tabular}

sition in the object, spatial variation in the detector response, gamma fogging, and statistical error in neutron detection in the high-absorption regions. Consequently, a formal calibration using a penetrameter is necessary to relate the tracer concentration and radiance. The penetrameter consists of two parallel aluminum plates with a known variable gap. The penetrameter is filled with the tracer solution to be imaged, and thermal neutron transmissivities are recorded as a function of the tracer thickness. Typical calibration curves for three fluids are shown in Figure 5. Deviations from exponential dependence appear to be prevalent when neutron absorption is high. Calibration curves can be used to determine the tracer concentration from measured values of the radiance.

\section{Radiographic Imaging of Reactive Dissolution}

Reactive dissolution of porous media is pertinent to many industrial processes, such as the stimulation of petroleum reservoirs, geological processes, and coal gasification. The first imaging study focused on reactive dissolution of oil-bearing porous media. In this process, called acidization, acids are injected into the formation to increase the flow conductivity of the near well-bore region in a petroleum reservoir. The majority of the world's oil is deposited in either sandstone or limestone formations, and acidization of these porous media can be divided into two categories depending on the mechanism of acidization. In the case of sandstone acidization near am-

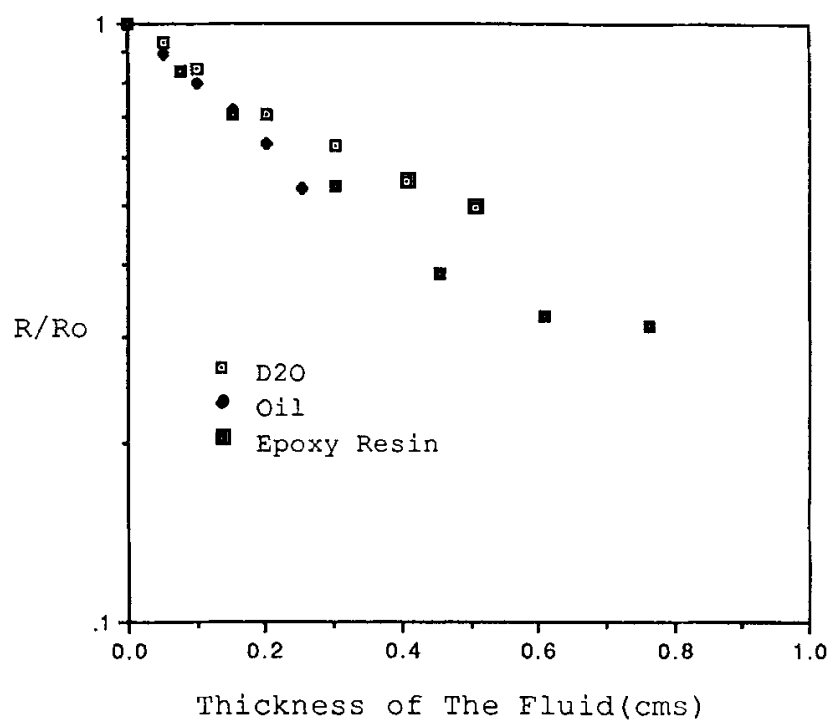

Figure 5. Neutron transmission curves for three fluids. 
bient temperatures, the reaction between the porous matrix and the acid is reaction-rate-limited and the reaction front moves uniformly through the porous medium (Lund and Fogler, 1976). In limestone acidization, however, the dissolution of the rock matrix by acid is mass-transfer-rate-limited (Hoefner and Fogler, 1987). The structure of dissolution patterns resembles root systems in plants, since it originates from the acid injection face and grows and branches out randomly away from the injection face. The dominant flow channel generated by acidization is called a wormhole. The diffusion-limited dissolution of limestone by hydrochloric acid is the focus of this study.

Experimental investigations of the reactive dissolution of porous media can be carried out using the Hassler apparatus. The experimental procedures are described in detail by Hoefner and Fogler (1987) and some salient features are summarized here. A limestone core, having a diameter of $3.75 \mathrm{~cm}$ and a length of $12.5 \mathrm{~cm}$, is placed in a Hassler cell, and the initial flow conductivity of the porous matrix is measured by flowing a nonreacting fluid through the core. At the onset of the experiment, acid is injected into the porous core at a known flow rate and the pressure drop across the system is monitored as a function of time. The pressure of the effluent fluid is maintained at $6,900 \mathrm{kPa}$ to keep gaseous reaction products in solution. The experiment is halted when wormholes reach the exit face of the porous matrix as evidenced when a negligible pressure drop occurs across the core.

The structure of the dissolution patterns can be identified by injecting molten Woods alloy into the acidized porous matrix. The injection pressure of the Woods metal can be controlled so that it can only invade the flow paths enlarged by acid. Previously, the porous limestone core was completely dissolved in acid leaving the solidified Woods metal to visualize the structure of the wormhole. It was found, however, that delicate branches of the porosity pattern would break during either the dissolution step or subsequently during the handling of the objects. Neutron radiography overcomes these limitations since the cadmium in Woods metal provides a high contrast in the neutron transmission images. Thermal neutron radiographs are obtained by exposing the Woods metal-filled porous media for $30 \mathrm{~s}$.

A neutron radiograph of a pattern generated at large Damköhler numbers is shown in Figure 6. A limestone core was acidized with 1 normal hydrochloric acid at an acid injection rate of $40 \mathrm{~mL} / \mathrm{h}$. The main characteristic of the dissolution pattern is the presence of a single dominant wormhole. The structure of dissolution patterns evolved during acidization is a function of the local flow rate and the rate of acid diffusion to the surface of the solid phase (Hoefner and Fogler, 1988). Effects of the convection and acid diffusion on the dissolution of the porous matrix can be quantified using a Damköhler number in the following manner:

$$
D a=\frac{\text { acid diffusion rate }}{\text { acid convection rate }}
$$

At very large Damköhler numbers, the acid dissolves the rock completely starting from the inlet face, and the size of the wormhole will be the same as that of the porous medium. As the Damköhler number is reduced, the porosity patterns become increasingly branched.

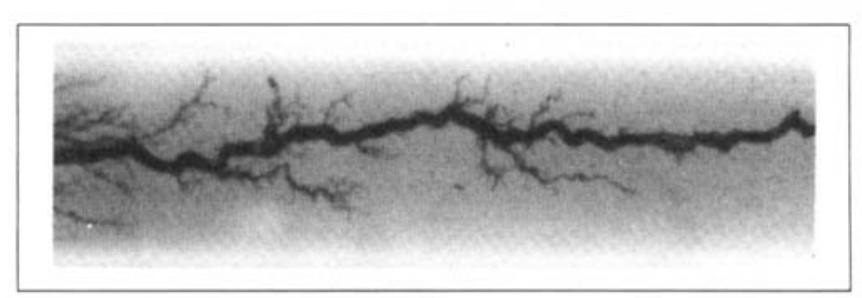

Figure 6. Porosity pattern resulting from dissolution at large reaction Damköhler number.

$\mathrm{HCl}$ injection rate: $40 \mathrm{~mL} / \mathrm{h}$.

The structure of the dissolution patterns is also influenced by the precipitation rate of reaction products. At sufficiently slow injection rates, the unconsumed acid front moves very slowly relative to the formation of reaction products. The precipitate formed by reaction products moves ahead of the reaction front and alters the flow profiles by plugging flow paths. The effect of precipitate on local flow profiles can be quantified using a Damköhler number for precipitation. It can be defined in a manner similar to the reaction Damköhler number where the precipitation rate is used in the numerator of Eq. 5. During the presence of both dissolution and precipitation phenomena, the overall conductivity of the porous medium experiences transient oscillations owing to the competing and opposite effects of both the dissolution and subsequent precipitation phenomena. The transient effects are detailed by Rege and Fogler (1989).

A neutron radiograph of porosity pattern generated at large precipitation Damköhler numbers and large dissolution Damköhler numbers is shown in Figure 7. The pattern in Figure 7 was generated by injecting 0.75 molar ferric chloride solution into the limestone matrix at the flow rate of $40 \mathrm{~mL} / \mathrm{h}$. The reactant has an initial $\mathrm{pH}$ of 1.5 and forms iron hydroxide when the solution $\mathrm{pH}$ rises owing to the reaction with the carbonate. The reaction product precipitates out of the liquid phase when the $\mathrm{pH}$ of the solution increases beyond 3. Figure 7 shows that the channels are highly tortuous and in some instances they propagate against the direction of mean flow. The local flow diversion is caused by the precipitation of iron hydroxide. Channel propagation in the direction opposite to the applied mean pressure gradient was unexpected and reinforces the need for flow visualization tools to understand complex flow phenomena in porous media. Owing to the fineness of the wormhole structure, these channels and this "backtracking" could have not been observed by other techniques to image the etched channels. A comparison of Figures 6 and

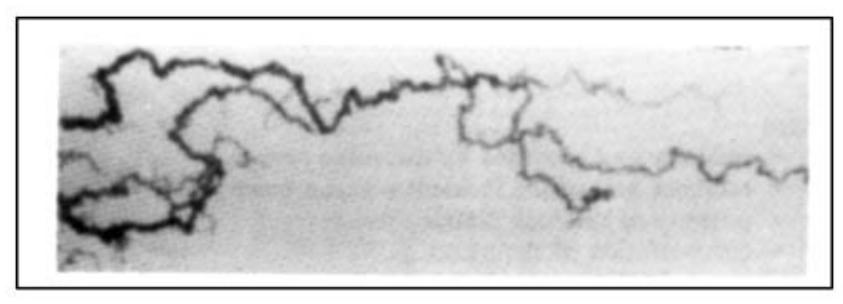

Figure 7. Porosity pattern resulting from the dissolution of limestone at large reaction and precipita. tion Damköhler numbers.

$\mathrm{FeCl}_{3}$ injection rate: $40 \mathrm{~mL} / \mathrm{h}$. 


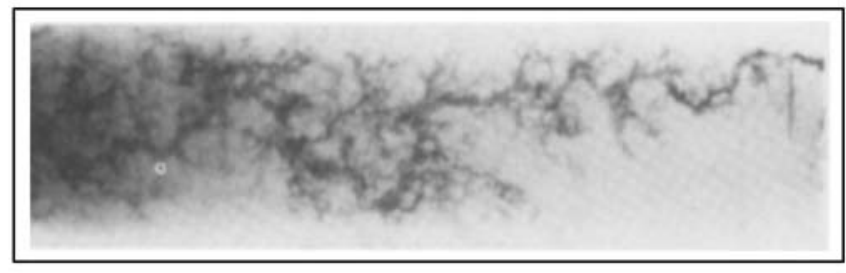

Figure 8. Porosity pattern generated at small reaction Damköhler number and large precipitation Damköhler number.

$\mathrm{FeCl}_{3}$ injection rate: $100 \mathrm{~mL} / \mathrm{h}$.

7 clearly shows the effect of precipitation on the wormhole structure. The $\mathrm{HCl}$ system does not form any precipitates and therefore the porosity patterns are less tortuous.

At very small precipitation Damköhler numbers, the concentration of reaction products is too low to cause precipitation from solution. The structure of wormholes generated in this case would depend only on the dissolution Damköhler number. An acid-etched pattern, generated by using ferric chloride at $100 \mathrm{~mL} / \mathrm{h}$ injection rate, is shown in Figure 8 . The resulting porosity pattern under these conditions is highly branched and is similar to the patterns generated with $\mathrm{HCl}$ at high flow rates (Hoefner and Fogler, 1986). The technique used by Hoefner and Fogler to visualize the acid-etched porosity pattern has two limitations. It is a destructive method where the entire porous matrix was dissolved away at the end of the experiment. Some of the finer branches of the pattern broke during the dissolution of the porous matrix. However, neutron imaging overcomes these limitations.

Images with greater spatial resolution can be obtained by

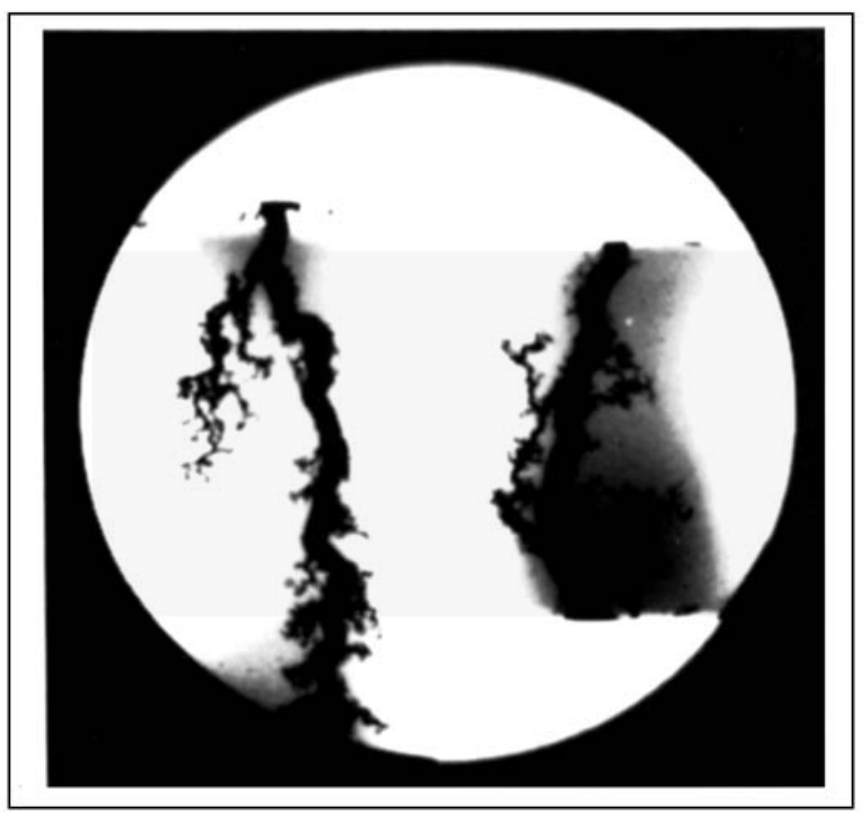

Figure 9. High-resolution neutron radiograph of a wormhole generated at large reaction Damköhler number.

Exposure time, $20 \mathrm{~min}$; a $25-\mu \mathrm{m}$-thick gadolinium metal layer used as the converter. using very thin converters and increasing the degree of collimation of the neutron beam. The path length of the actinic radiation generated by the converter increases as the thickness of the converter is increased. The degree of blurring of an image increases with the thickness of the converter since the probability of the deflection of secondary radiation increases (Barton and von der Hardt, 1981). An example of a higherresolution image obtained using a thinner screen is shown in Figure 9, where the image was generated using narrower and a well-collimated beam of thermal neutrons. Because the diameter of the collimator is smaller than the length of the porous core, the core was divided into two pieces. A vapor-deposited gadolinium metal layer having a thickness of $25 \mu \mathrm{m}$ is used as a converter that generates electrons upon absorbing thermal neutrons. Higher-resolution images require longer exposure times since more neutrons pass through a thin screen instead of being absorbed by it. The image in Figure 9 was obtained with an exposure time of $20 \mathrm{~min}$. These results clearly establish the effectiveness of film neutron radiography in imaging the porosity patterns generated during the acidization process.

\section{Application of Real-Time Radiography to Miscible Flow}

Capabilities of the real-time neutron imaging system were demonstrated using the well-known miscible dispersion phenomenon. Miscible flow in porous media is of fundamental interest to a wide variety of processes, such as secondary and tertiary oil recovery processes, salt water intrusion in coastal aquifers, contaminant transport in groundwater, and packedbed processes. Processes of dispersion and mixing two miscible fluids in porous medium have been extensively studied (Blakwell, 1962; Perkins and Johnston, 1963; Fried and Combarnus, 1971; Jerauld, 1985 and its references). The combined action of local velocity gradients and molecular diffusion dissipate an initial sharp front between two miscible fluids. The mixing process in a homogeneous porous medium can be simulated using a convective-dispersion equation in which the spatial distribution of a tracer pulse injected in a porous medium can be approximated using a normal distribution function.

The time evolution of a miscible tracer pulse was imaged using the real-time thermal neutron imaging system. A Berea sandstone slab having dimensions of $1.9 \mathrm{~cm} \times 7.6 \mathrm{~cm} \times 20.3$ $\mathrm{cm}$ was used for the experiments. Two aluminum distributor caps having dimensions of $1.9 \mathrm{~cm} \times 7.6 \mathrm{~cm}$ were placed on the sides of the Berea slab to allow the injection and withdrawal of the fluids. The distributors and the slab were sealed using a 0.25 -cm-thick layer of white epoxy. Deuterium oxide was used as the solvent and $4 \%$ sodium borate solution in deuterium oxide was used as the tracer. Boron provides excellent contrast over both the porous matrix and the solvent due to its large thermal neutron cross-section. At the beginning of each experiment, 0.05 pore volume of the tracer was injected into the porous matrix and neutron images were recorded on a video tape as the tracer traversed through the porous matrix.

Figure 10 shows three transmission images following the advancement of the miscible tracer pulse through Berea sandstone slab. The solvent is injected at a flow rate of $360 \mathrm{~mL} / \mathrm{h}$. The time lag between the first and the second image is approximately 7 min. Transmission images in Figure 10 are the imprints of color-coded images. In the original color-coded images, the 


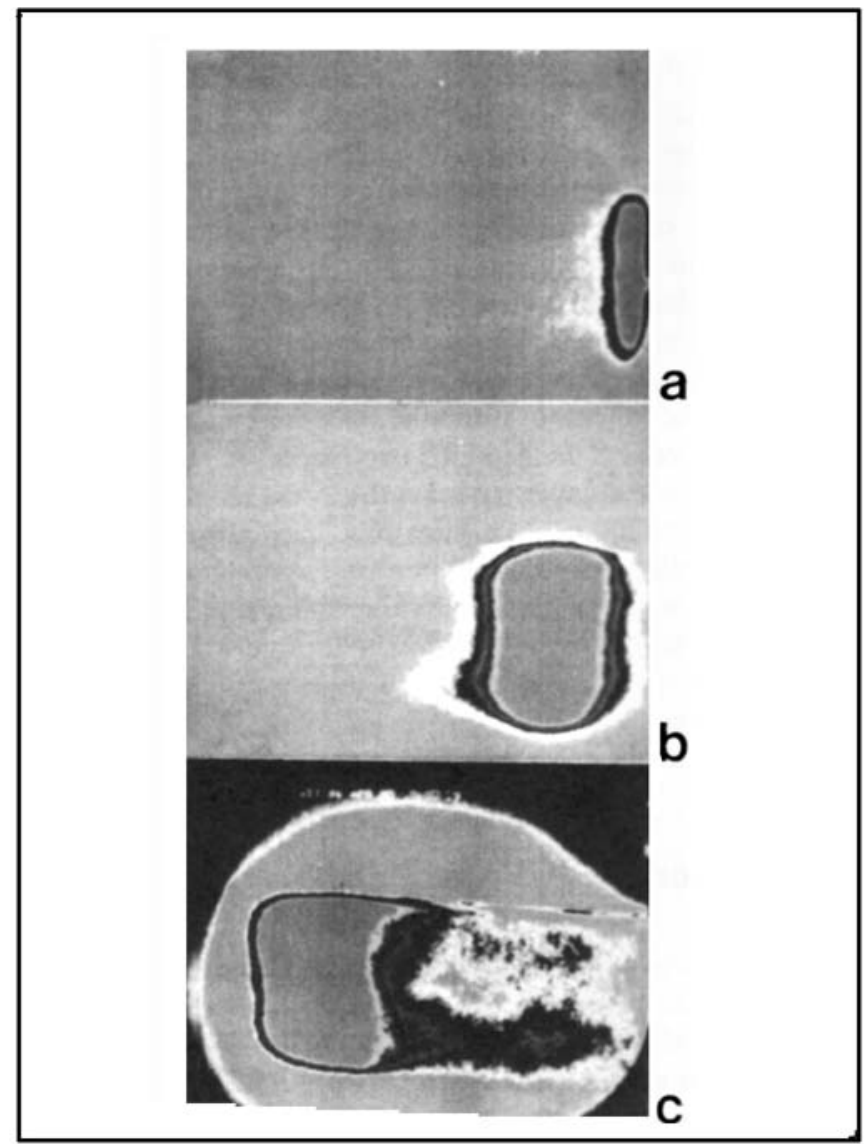

Figure 10. Neutron images of miscible tracer dispersion.

tracer pulse was shown in blue whereas the background was in red. Unfortunately, both colors show similar gray tones when reproduced in black and white in Figure 10. However, one can clearly identify the tracer since the transition colors representing low tracer concentration regions provide a contrast in the black and white image. Figure 10a shows the tracer pulse immediately upon its entry into the porous matrix. A comparison of the width of the tracer pulse in the three images show that the width of the tracer pulse initially increases dramatically, but the rate of growth appears to be negligible as the tracer reaches the exit of the porous slab. This is not unexpected since dispersion is a diffusive phenomenon, where the standard deviation of the tracer concentration distribution increases with the square root of time.

Neutron transmission images stored on a high-resolution video tape were digitized into an array of $640 \times 480$ pixels. The images were averaged over 200 successive video frames to remove temporal noise. Tracer concentrations were measured using the calibration procedures discussed above as shown in Figure 11 for an experiment performed at $360-\mathrm{mL} / \mathrm{h}$ injection rate. Each point was obtained by averaging the neutron transmissivity in the transverse direction. The tracer distribution follows the expected Gaussian profile, and the standard deviation of each profile increases with time owing to the mixing process. Some degree of skewness is observed in the profiles taken beyond the injection of 0.3 pore volume of the solvent. The skewness is caused by a small fraction of the pore space

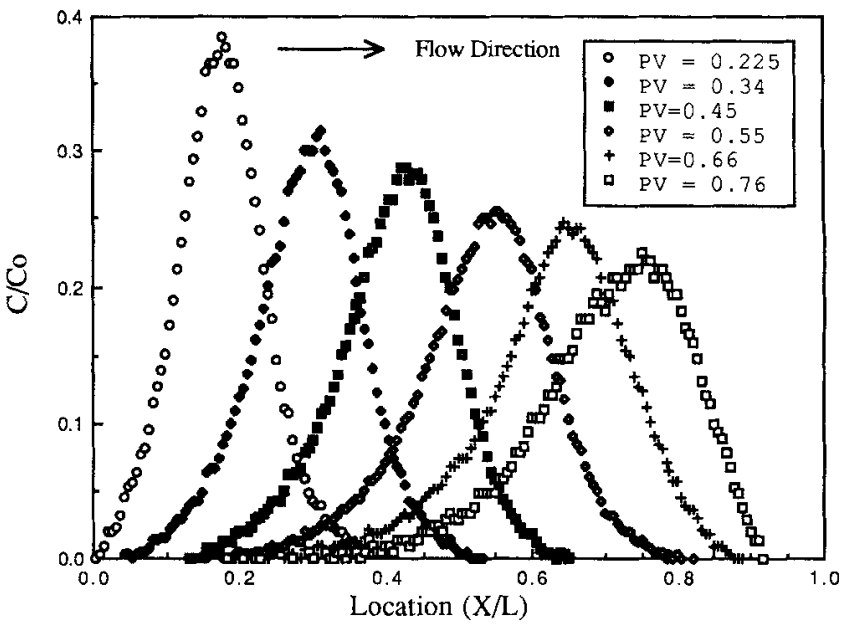

Figure 11. In-situ concentration profiles obtained from digitized images.

It illustrates the process of dispersion in sandstone.

having a very low flow conductivity. Tracer transport through this region is diffusion-limited, and the time scale for tracer transport in this region would be greater than the convective time scale.

An interesting effect is observed when the flow direction is reversed during the course of a dispersion experiment. The spread in the spatial distribution narrows initially for a period of time when the flow direction is reversed. This effect is shown in Figure 12, where the square of the normalized standard deviation of the spatial distribution of the tracer is shown as a function of the pore volumes of the fluid injected. The flow direction was reversed after injecting 0.75 pore volume of the solvent in the forward direction. The standard deviation of the tracer decreases upon flow reversal for a brief period of time during which the local concentration of the tracer increases. The tracer again undergoes the typical dilution process after this period.

Dispersion is a result of local variations of flow conductances

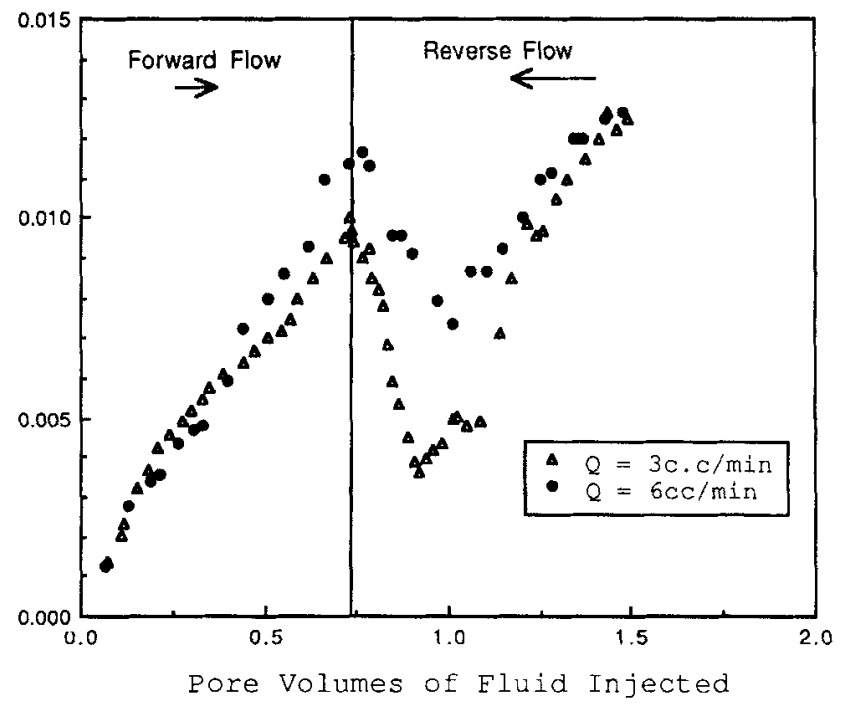

Figure 12. Effect of flow reversal on the standard deviation of the tracer spatial distribution. 


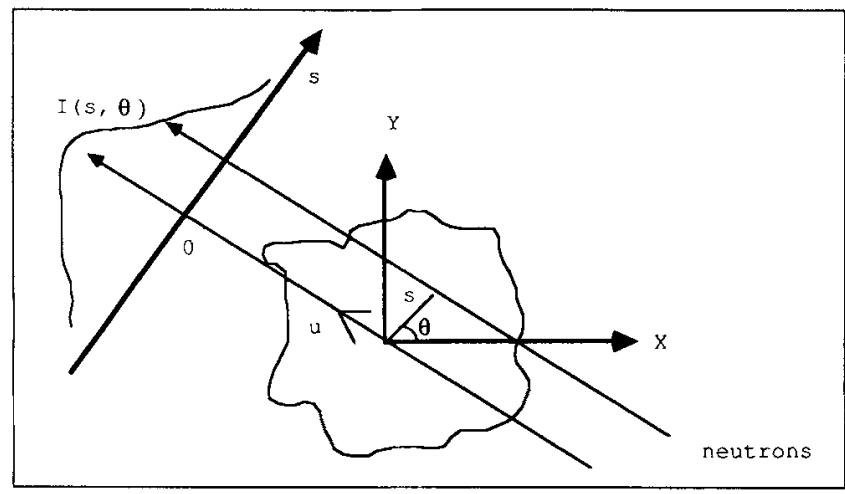

Figure 13. Neutron transmission for a two-dimensional object.

The transmissivity $I$ is related to the attenuation of neutron beam along its path length $u$.

in a porous medium and molecular diffusion. Variations in local velocity profiles are caused by the randomness in the microstructure of the porous medium. At any given time, tracer molecules present at the forefront of the tracer pulse represent faster flow paths. Conversely, tracer molecules lagging behind the mean location of the tracer distribution represent slow flow paths. Tracer molecules appear to retrace their flow paths for a brief time period when the flow is reversed, and as a consequence, the tracer pulse undergoes a concentrating process. This phenomenon was first discussed by Heller (1972) and was referred to as "unmixing." His experimental results were qualitative, whereas our in-situ tracer concentrations and standard deviation measurement of the tracer concentration profiles in naturally occurring porous media are presented for the first time.

\section{Computer Tomography}

Computer tomography (CT) has the advantage of providing a three-dimensional description of an object. Although basic principles of CT are well known, some salient features are reproduced here for the sake of completeness. $\mathrm{CT}$ involves the

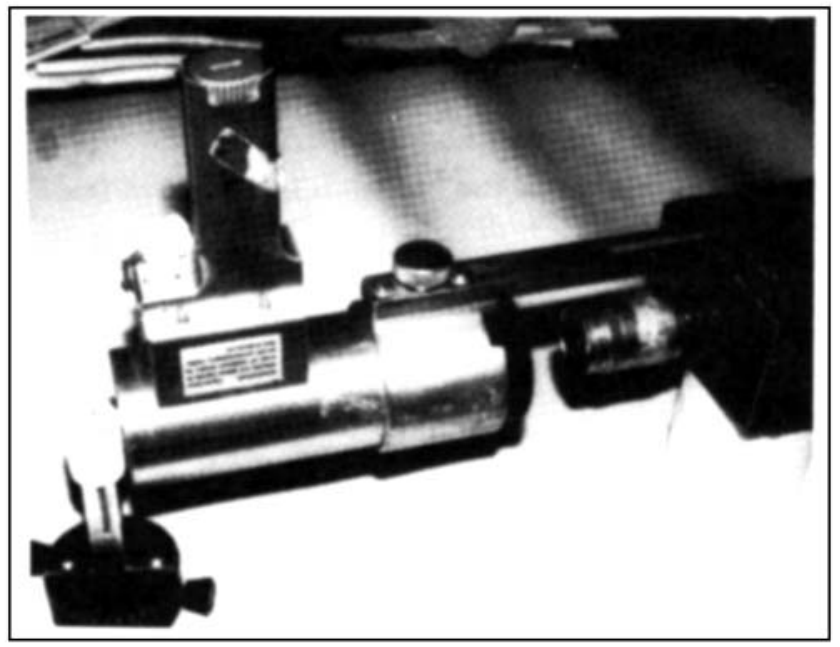

Figure 14. Components of the neutron tomography apparatus. reconstruction of a three-dimensional property of an object from the measurement of several two-dimensional transaxial projections. For the case of neutron CT, the neutron linear attenuation coefficient is computed. This concept is shown in Figure 13 for a two-dimensional case. The object to be reconstructed is placed in the path of a two-dimensional thermal neutron beam. The transmission image is captured using either the film method or the real-time method.

$$
I(s, \theta, z)=I_{0} \exp \left[-\int_{0}^{1} \mu_{0}(x, y, z) d u\right]
$$

where

$\mu_{o}=$ linear attenuation coefficient, $\mathrm{cm}^{-1}$

$l=$ pathlength, $\mathrm{cm}$

$u=$ dummy variable in Figure 13, $\mathrm{cm}$

Several transmission images are obtained by rotating the object about its axis. Image reconstruction can be accomplished by solving the integral equation for $\mu$, if the transmission values are known. Because it is solved on a discretized mesh, the solution procedure is called computer tomography.

Figure 14 shows the important components of the CT apparatus. A collimator with a length to diameter ratio of 340 was used to generate a near-parallel beam of thermal neutrons. The acidized limestone core filled with Woods metal in Figure 9 was used for tomographic reconstruction. This core was mounted on to a manual rotator with an aluminum fixture to obtain 60 transmission images at $3^{\circ}$ angular rotation intervals. Each projection image was digitized using the image-processing system. Together, all the transmission images constitute the array: I (512, theta, 480$)$ where the value of theta varies from 0 to 177 in steps of $3^{\circ}$. The intensity values are normalized using the transmission values recorded from the regions unobstructed by the object. Computer reconstructions are performed using the filtered-back projection algorithm (Jain, 1989).

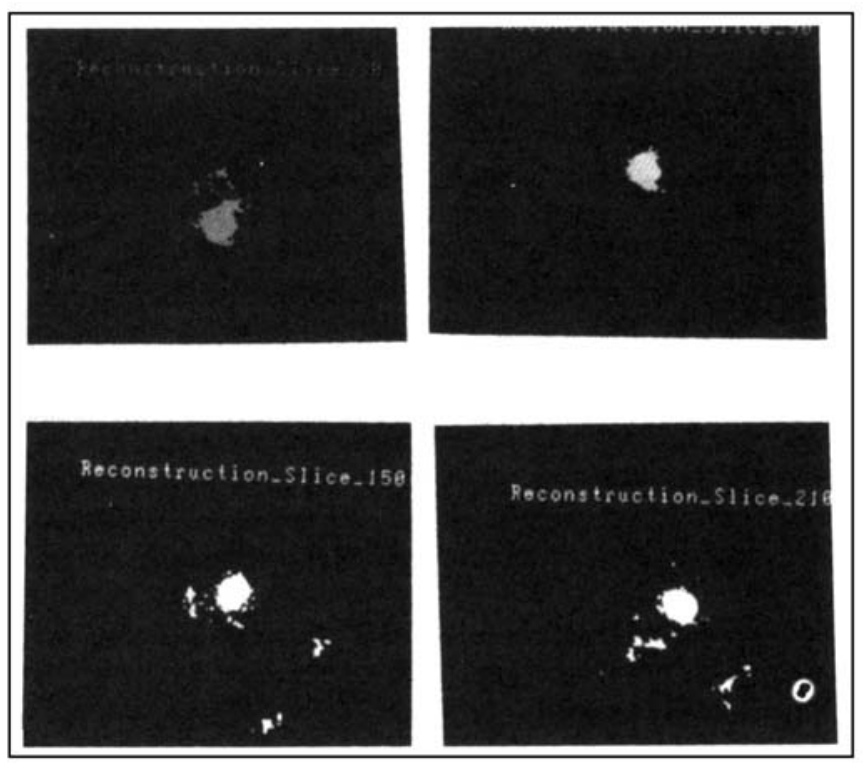

Figure 15. Tomographic reconstructions of an acidized carbonate. 
Binary images of two-dimensional slices from the reconstructed data set are shown in Figure 15. Regions having large thermal neutron cross-sections are shown in white, representing the Woods metal. The images are transaxial views and different branches appear as isolated blobs. CT allows full description of the object and facilitates the measurement of any desired property. In the case of acid-etched porosity patterns, one can measure the volume fraction, surface area and tortuosity of the wormholes. It is possible to directly correlate the tortuosity with the Damköhler number for precipitation. Similarly, one would expect a correlation between the specific surface area and the reaction Damkohler number. The CT system is currently being upgraded with automated rotator/translator to make such measurements available with minimal effort.

\section{Conclusions}

- Thermal neutron transmission radiography is ideally suited for imaging various flow phenomena in natural porous media. The power of this technique relies on the fact that elements present in most of the porous media possess very small thermal neutron cross-sections. Elements such as gadolinium, cadmium, boron, and hydrogen provide high contrast in the transmission images. These elements can be used as tracers in a fluid component to be imaged.

- Our results on acidization show that the structure of the acid-etched porosity patterns are influenced strongly by the value of the Damköhler number for the precipitation of reaction products. Precipitation of reaction products results in highly tortuous wormholes where flow can be diverted against the direction of the mean pressure gradient. This "backtracking" effect has not been observed by other imaging techniques.

- Capabilities of real-time neutron imaging have been demonstrated using the well-known miscible dispersion process. Flow processes with a time resolution of seconds can be imaged with real-time neutron radiography. In addition to providing visual diagnostic information, it is possible to measure in-situ quantitative information using neutron imaging.

- Neutron computer tomography can be used to obtain threedimensional information. Potential application of CT includes the measurement of tortuosity and connectivity of the acidetched patterns.

\section{Acknowledgment}

The authors acknowledge the members of the porous media Industrial Affiliates program at The University of Michigan for financial support. Member companies are: Chevron, Conoco, Halliburton, Japan National Oil Corp., Marathon, Mobil, Phillips Petroleum, Texaco, and UNOCAL.

\section{Notation}

$C=$ concentration of tracer in the solvent, $\mathrm{g} / \mathrm{cm}^{3}$

$D a=$ Damköhler number, dimensionless

$I=$ thermal neutron flux passing through the object, no. $/ \mathrm{cm}^{2} \cdot \mathrm{s}$

$I_{0}=$ unattenuated thermal neutron flux, no. $/ \mathrm{cm}^{2} \cdot \mathrm{s}$

$l=$ path length, $\mathrm{cm}$

$R=$ radiance units recorded by the detector at the location on the object

$R_{0}=$ radiance units recorded by the detector at a location where no attenuation of the neutron beam occurs

$w_{j}=$ weight fraction of an element in a compound

$x=$ thickness of the object, $\mathrm{cm}$

\section{Greek letters}

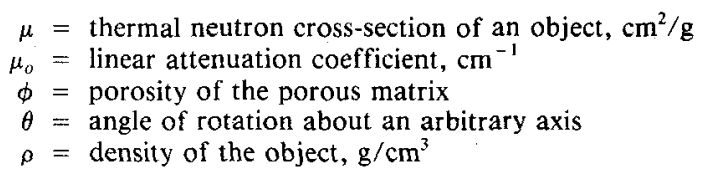

\section{Literature Cited}

Barton, J. P., and P. von der Hardt, Neutron Radiography Handbook, D. Reider Publishing, Boston (1981).

BlakwelI, R. J., "Laboratory Studies of Microscopic Dispersion Phenomena," Soc. Pet. Eng. J., 2, 1 (1962).

Fried, J. J., and M. A. Combarnus, "Dispersion in Porous Media," Adv. in Hydro. Sci., 7, 169 (1971).

Hawkesworth, M. R., and J. Walker, "Basic Principles of Thermal Neutron Radiography," Neutron Radiography, p. 5, Proc. World Conf., J. P. Barton and P. von der Hardt, eds. (1983).

Heller, J. P., "Observations of Mixing and Diffusion in Porous Media," Proc. Symp. on Fundamentals of Transport Phenomena in Porous Media, Int. Assoc. for Hydraulic Res. Committee, Int. Soc. of Soil Sci. Commission, Univ. of Guelph (Aug. 7-16, 1972).

Hoefner, M. L., and H. S. Fogler, "Pore Evolution and Channel Formation During Flow and Reaction in Porous Media," AIChE $J ., 34(1), 45$ (1988).

Hoefner, M. L., and H. S. Fogler, "Effect of Acid Diffusion in Matrix Acidizing in Carbonates," J. Pet. Tech., 39, 203 (1987).

Jain, A. K., Digital Image Processing, Prentice Hall (1989).

Jerauld, G. R., "Flow and Transport in Chaotic Media: Four Case Studies," PhD Thesis, Univ, of Minnesota, Minneapolis (1985).

Lund, K., and H. S. Fogler, "Acidization: V. The Prediction of the Movement of Acid and Permeability Fronts in Sandstone," Chem. Eng. Sci., 31, 381 (1976).

Perkins, T. K., and G. C. Johnston, "A Review of Diffusion and Dispersion in Porous Media," Pet. Trans. AIME, 228, 77 (1963).

Rege, S. D., and H. S. Fogler, "Competition among Flow, Dissolution Precipitation in Porous Media," AIChE J., 35(7), 1177 (1989).

Wellington, S. L., and H. J. Vinegar, "Tomographic Imaging of Three-Phase Flow Experiments," Rev. Sci. Instruments, 58, 96 (Jan., 1987).

Manuscript received Nov. 12, 1990, and revision received Jan. 17, 1992. 